\title{
Densidad básica, longitud de fibras y crecimiento en dos procedencias de Eucalyptus globulus en Argentina
}

\author{
Basic wood density, fiber length and growth rate in two \\ Eucalyptus globulus provenances from Argentina
}

\author{
Dora Igartúa ${ }^{a^{*}}$, Silvia Monteolivab

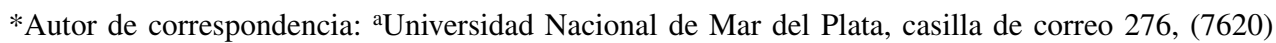 \\ Balcarce, Argentina, digartua@balcarce.inta.gov.ar \\ bUniversidad Nacional de La Plata, La Plata, provincia de Buenos Aires, Argentina.
}

\begin{abstract}
SUMMARY
The aims of the work were to assess basic wood density and fiber length variation in two provenances of Eucalyptus globulus growing in Buenos Aires, Argentine, and to analyze the relationships between wood properties and growth rate. Seven years old trees were selected, 10 corresponding to local and 10 to Valdivia-Chile provenances. The wood properties were determined at different tree heights and at three radial positions for the fiber length. The provenances were significantly different in basic density (local $546.9 \pm 36 \mathrm{~kg} \mathrm{~m}^{-3}$; Valdivia-Chile $490.8 \pm 38 \mathrm{~kg} \mathrm{~m}^{-3}$ ) and similar in fiber length (local $863.6 \pm 38.8 \mu \mathrm{m}$; Valdivia-Chile $830.2 \pm 67.4 \mu \mathrm{m})$. The analyses of variance showed the importance of sample height for basic density variation, and radial position for fiber length. The provenances had different tendencies across the log for wood density. The local provenance presented increasing wood density from the base to the top; however Valdivia-Chile provenance had not variation. Fiber length decreased slightly to the top in both local and Valdivia-Chile provenances. The random variance of tree factor was very important for density (62\%) and for fiber length $(59 \%)$. Provenances had different total height increment (Valdivia-Chile $2.6 \pm 0.3 \mathrm{~m}^{2}$ year ${ }^{-1}$, local $2.1 \pm 0.2 \mathrm{~m}$ year $\left.^{-1}\right)$. No correlation was present between growth rate and wood properties.
\end{abstract}

Key words: wood quality, wood properties, within and between tree variability, growth rate.

\section{RESUMEN}

Los objetivos del presente trabajo fueron evaluar la variación de la densidad básica y la longitud de fibras de la madera de dos procedencias de Eucalyptus globulus implantadas en la provincia de Buenos Aires, Argentina. Se analizó además la relación entre estas propiedades de la madera con los crecimientos de los árboles. Se estudiaron árboles de siete años de edad, 10 de procedencia local y 10 de Valdivia-Chile. Se determinaron la densidad básica y la longitud de fibras a diferentes alturas en el fuste y en tres posiciones radiales para la longitud de fibras. Se encontraron diferencias significativas para la densidad (local $546,9 \pm 36 \mathrm{~kg} \mathrm{~m}^{-3}$; Valdivia-Chile 490,8 $\pm 38 \mathrm{~kg} \mathrm{~m}^{-3}$ ), pero no para la longitud de fibras (local 863,6 \pm 38,8 $\mu \mathrm{m}$; Valdivia-Chile 830,2 $\pm 67,4 \mu \mathrm{m}$ ) Asimismo, se encontraron diferencias significativas con la altura de muestreo para la densidad y para la longitud de fibras, y con la posición radial para longitud de fibras. A lo largo del fuste las procedencias manifestaron patrones diferentes de variación para la densidad: la local, aumentó en sentido base-ápice y Valdivia-Chile mostró ausencia de variación; y tendencias de descenso leve base-ápice para la longitud de fibras. La varianza debida a árboles fue de $62 \%$ para la densidad y $59 \%$ para la longitud de fibras. Las procedencias se diferenciaron sólo por su incremento medio anual en altura (Valdivia-Chile 2,6 m año ${ }^{-1} \pm 0,3 \mathrm{~m}$ año-1, local $2,1 \mathrm{~m}$ año ${ }^{-1} \pm 0,2 \mathrm{~m}$ año ${ }^{-1}$, no encontrándose correlaciones entre crecimientos medios y las propiedades de la madera.

Palabras clave: calidad de madera, propiedades de la madera, variabilidad dentro y entre árboles, crecimiento medio.

\section{INTRODUCCIÓN}

La madera de Eucalyptus globulus Labill. es una de las mejores para la elaboración de pulpas de fibra corta para papeles de escritura de alta calidad. La delignificación, el refino y el blanqueo se logran con buenos rendimientos y bajo consumo de productos químicos (Torres y Rodríguez 1991, Valente et al. 1992). Entre los atributos de la pulpa se mencionan la lisura, la opacidad, la porosidad y las propiedades de drenaje y de secado de la hoja de papel, lo que le otorga excelencia para la manufactura de papeles de impresión, escritura y tissue (Foelkel 1998).

El litoral marítimo del sudeste de la provincia de Buenos Aires-Argentina representa una buena condición de sitio para el desarrollo de Eucalyptus globulus (Golfari 1957, Moschini et al. 2000). Las masas regionales se han implantado con dos procedencias geográficas de la especie, la local y Valdivia-Chile. Esta última ha sido 
elegida por algunos asesores forestales a partir del año 1994 con base en criterios que no se han hecho públicos ni responden a políticas de promoción estatal (Igartúa y Monteoliva 2006).

Este recurso forestal ha sido implantado con el objetivo de proveer materia prima fibrosa para la industria celulósico-papelera, pero no ha sido evaluado en los aspectos dasométricos ni en relación con la calidad de su madera para tal uso. La densidad básica es la más importante propiedad de la madera para la evaluación de la calidad de los productos fibrosos (Zobel 1997); ha probado ser un apropiado estimador de la calidad para la elaboración de pulpas asociándose a los rendimientos, volumen específico y resistencias en pulpas Kraft (Valente et al. 1992, Ramírez et al. 2009). Otro atributo, la longitud de las fibras, es también considerado en la evaluación de la aptitud papelera de la madera ya que está asociado con el logro de diferentes características de los papeles en términos de sus resistencias, propiedades ópticas y características superficiales (Foelkel 1998).

El estudio de estos atributos de la madera ha indicado la existencia de variaciones dentro y entre árboles. En latifoliadas de porosidad difusa, la variación entre árboles, resultante de causas genéticas y medioambientales, es considerada por algunos autores como la mayor fuente de variación en la densidad, en tanto que la variación dentro del árbol relacionada con las diferentes edades formativas del leño lo es para la morfología de las fibras. Así lo expresan Downes et al. (1997) en su revisión sobre modelos o patrones de diferentes propiedades del leño en el género Eucalyptus y sus fuentes de variación; y Kojima et al. (2009) en su revisión sobre los modelos de maduración de fibras en latifoliadas de rápido crecimiento.

En el sudeste de la provincia de Buenos Aires, Argentina, se han llevado a cabo estudios locales relativos a la caracterización anatómica, física y química de la madera de E. globulus implantado en cortinas forestales y masas de reparo para el ganado (Igartúa et al. 2000, 2002, 2003) y en plantaciones comerciales (Igartúa y Monteoliva 2006). Pero hasta el momento no se ha abordado el estudio de la variabilidad que, dentro de las descripciones generales para el género o la especie, puede manifestarse entre las dos procedencias utilizadas comercialmente, lo que se espera sea un conocimiento de interés frente al consumo de esta materia prima por el sector industrial y frente a las decisiones de implantación de futuras masas comerciales. Tampoco se ha analizado la relación entre las propiedades de la madera y los crecimientos manifestados localmente, desconocidos debido a la ausencia de inventarios locales exhaustivos y sistemáticos. Los objetivos del presente trabajo son evaluar la variabilidad entre las procedencias como también entre y dentro de los individuos, de la densidad básica y la longitud de fibras de la madera de Eucalyptus globulus, y analizar la relación entre estas propiedades de la madera y los crecimientos de los árboles individuales.

\section{MÉTODOS}

El material experimental correspondió a individuos de dos plantaciones comerciales de E. globulus de siete años de edad, una de procedencia local y otra de procedencia Valdivia, Chile (Valdivia-Chile). Las masas fueron implantadas en un establecimiento del Partido de Necochea (Provincia de Buenos Aires, Argentina), con similares distanciamientos iniciales (2.000 árboles ha ${ }^{-1}$ ) y tratamientos de implantación, no realizándose ninguna intervención silvicultural ni evaluación dasométrica durante su desarrollo. Los árboles estudiados tuvieron una altura total promedio de $14,4 \pm 1,1 \mathrm{~m}$ (procedencia local) y $18,3 \pm 2,1 \mathrm{~m}$ (procedencia Valdivia-Chile); en tanto que sus diámetros promedio a la altura del pecho fueron, respectivamente, $15,9 \pm 0,9 \mathrm{~cm}$ y $15 \pm 2,6 \mathrm{~cm}$.

Estudio de la densidad básica y la longitud de fibras. Se seleccionaron 10 árboles al azar por cada procedencia sobre los que se practicó un muestreo destructivo, tomándose tres rodajas a cada una de cinco alturas de muestreo a lo largo del fuste: la base (0,3 m sobre el nivel del suelo), la altura de pecho a 1,3 m sobre el nivel del suelo (AP) y el $30 \%$, $50 \%$ y $75 \%$ de la altura total. Dos rodajas por altura de muestreo fueron cortadas por la mitad y descortezadas para las determinaciones de la densidad básica (peso seco en estufa/volumen verde) según la norma TAPPI T 254-om94. Las semirrodajas se utilizaron completas. En cuatro de las cinco alturas de muestreo (base, AP, $30 \%$ y $50 \%$ de la altura total) y en tres posiciones radiales $(10 \%$, $50 \%$ y $90 \%$ de la longitud del radio), la tercera rodaja se destinó al estudio de la longitud de fibras midiéndose previamente la longitud de sus radios norte-sur y este-oeste. La determinación de la longitud de fibras se realizó sobre macerados según la técnica de Franklin (Jane 1970). Se tomaron una o dos astillas correspondientes a un segmento de radio de $0,5 \mathrm{~cm}$ de longitud. Tal segmento se definió centrado en la posición de muestreo, tanto en el radio de orientación norte como en el de orientación sur. Se midió la longitud de 60 fibras al azar por cada posición radial mezclando, para cada posición, los macerados de los puntos cardinales norte y sur. Las mediciones se realizaron en un microscopio Olympus con analizador de imágenes utilizando un aumento de $4 \mathrm{X}$.

Análisis de la relación entre parámetros de calidad de la madera y crecimiento. La estimación de los parámetros correspondiente al árbol completo se resolvió como el valor medio entre las estimaciones en cada altura (y en cada posición radial para el caso de longitud de fibras). Para la determinación de los incrementos medios anuales se consideró como fuste a la parte del tallo situada entre el tocón y la base de la copa, su altura y volumen se consideraron como altura y volumen comerciales. El diámetro normal o de referencia se definió a 1,3 m sobre el nivel del suelo (DAP) (Cailliez 1980). Se desarrolló la 
cubicación compuesta de los árboles aplicando la fórmula volumétrica de Smalian a cada una de las trozas de $2 \mathrm{~m}$ en que se seccionó el fuste. La sumatoria de los volúmenes de las trozas fue considerada como el volumen comercial real del árbol (en adelante, volumen comercial). El crecimiento o incremento medio anual (IMA) se calculó como la magnitud actual de las variables de interés en los individuos (DAP, altura total y volumen comercial) divididas por la edad actual en años de los mismos (Caillez 1980, Prodan et al. 1997).

Análisis estadísticos. Los datos de densidad básica y longitud de fibras fueron sometidos al análisis de varianza, tomando como fuentes de variación: procedencia, árbol, altura en el fuste y posición radial (esta última para el caso de la longitud de fibras). El análisis de la varianza se desarrolló bajo un modelo lineal mixto donde árbol se consideró un efecto aleatorio y el resto de las fuentes de variación como efectos fijos. El valor de probabilidad alfa establecido para calificar como significativas a las diferencias encontradas entre las fuentes de variación fue de 0,05. Los modelos estadísticos empleados para densidad básica y longitud de fibras, respectivamente, fueron los siguientes:

$$
\begin{gathered}
\mathrm{y}_{i j k}=\mu+\alpha_{\mathrm{i}}+\beta_{\mathrm{j}}+\delta_{\mathrm{k}(\mathrm{i})}+\alpha \beta_{\mathrm{ij}}+\varepsilon_{\mathrm{jk}} \\
i=1,2 j=1,2,3,4 k=1,2, \ldots 10
\end{gathered}
$$

donde:

$\mathrm{y}_{\mathrm{ijkl}}$ : densidad básica correspondiente al k-ésimo árbol en la j-ésima altura del fuste en la i-ésima procedencia,

$\alpha_{i}$ : efecto fijo de la i-ésima procedencia,

$\beta_{\mathrm{j}}$ : efecto fijo de la j-ésima altura del fuste,

$\delta_{\mathrm{k}(\mathrm{i})}$ : efecto aleatorio del k-ésimo árbol anidado en la iésima procedencia,

$\alpha \beta_{\mathrm{ij}}$ : efecto fijo de interacción entre i-ésima procedencia y j-ésima altura del fuste,

$\varepsilon_{\mathrm{jk}(\mathrm{i})}$ : efecto aleatorio del k-ésimo árbol en la j-ésima altura en la i-ésima procedencia (error aleatorio).

$\mathrm{y}_{i j k l}=\mu+\alpha_{\mathrm{i}}+\beta_{\mathrm{j}}+\gamma_{\mathrm{k}+} \delta_{1(\mathrm{i})}+\alpha \beta_{\mathrm{ij}}+\alpha \gamma_{\mathrm{ik}}+\beta \gamma_{\mathrm{jk}}+\varepsilon_{\mathrm{jkl}(\mathrm{i})}$ $i=1,2 j=1,2,3,4 k=1,2,3 l=1,2, \ldots 10$

Donde:

$\mathrm{y}_{\mathrm{ijkl}}$ : longitud de fibras correspondiente a la k-ésima posición radial en la j-ésima altura del fuste del l-ésimo árbol en la i-ésima procedencia,

$\alpha_{i}$ : efecto fijo de la i-ésima procedencia,

$\beta_{\mathrm{j}}$ : efecto fijo de la j-ésima altura del fuste,

$\gamma_{\mathrm{k}}$ : efecto fijo de la k-ésima posición radial,

$\delta_{1(\mathrm{i})}$ : efecto aleatorio del l-ésimo árbol anidado en la iésima procedencia,

$\alpha \beta_{\mathrm{ij}}$ : efecto fijo de interacción entre i-ésima procedencia $\mathrm{y}$ j-ésima altura del fuste,

$\alpha \gamma_{\mathrm{ik}}$ : efecto fijo de interacción entre i-ésima procedencia y k-ésima posición radial, $\beta \gamma_{\mathrm{j} k}$ : efecto fijo de interacción entre j-ésima altura del fuste y k-ésima posición radial,

$\varepsilon_{\mathrm{jkl}(\mathrm{i})}$ : efecto aleatorio del 1-ésimo árbol, de la i-ésima procedencia, en la j-ésima altura y k-ésima posición (error aleatorio).

Se desarrollaron pruebas de comparaciones múltiples de medias mediante la prueba de Tukey para abrir las interacciones. Para analizar la naturaleza de la relación entre los parámetros de calidad de la madera y el incremento medio anual de las variables de inventario (DAP, altura total y volumen comercial) se desarrolló una prueba de correlación (coeficiente de Pearson) (Kuhel 2003). Los análisis estadísticos se realizaron con el software R, de libre uso, versión 2.5.1 (R Development Core Team 2007).

\section{RESULTADOS}

Densidad básica. El valor promedio de la densidad básica de los 20 árboles analizados fue de $517,7 \mathrm{~kg} \mathrm{~m}^{-3}$ con un rango entre $608,4 \mathrm{~kg} \mathrm{~m}^{-3}$ y $423,6 \mathrm{~kg} \mathrm{~m}^{-3}$. La densidad promedio de la madera de la procedencia Valdivia-Chile fue menor $\left(490,8 \mathrm{~kg} \mathrm{~m}^{-3} \pm 38 \mathrm{~kg} \mathrm{~m}^{-3}\right)$ que la de la procedencia local $\left(546,9 \mathrm{~kg} \mathrm{~m}^{-3} \pm 36 \mathrm{~kg} \mathrm{~m}^{-3}\right)$ y de una mayor variabilidad entre árboles. El análisis de varianza mostró evidencia de la significancia de los factores procedencia $(P=0,0025)$ y altura $(P<0,001)$, y de la interacción entre ambos $(P<0,001)$. Por otra parte, la varianza aleatoria correspondiente al factor árbol resultó muy destacada dado que correspondió al $62 \%$ de la varianza aleatoria total.

Los contrastes de medias como apertura de la interacción procedencia-altura (cuadro 1) permitieron valorar como no significativas a las diferencias de densidad a lo largo del fuste en la procedencia Valdivia-Chile. La procedencia local manifestó una tendencia diferente por cuanto, a partir del $30 \%$ de la altura total, la densidad aumentó de manera significativa. Excepto a la altura del pecho, la densidad resultó siempre significativamente mayor en la procedencia local.

Cuadro 1. Variación de la densidad básica $\left(\mathrm{kg} \mathrm{m}^{-3}\right)$ según las alturas en el fuste y las procedencias. Promedios \pm desviación estándar. Prueba de Tukey, alfa $=0,05$.

Variation of basic density $\left(\mathrm{kg} \mathrm{m}^{-3}\right)$ by sample height and provenances. Mean \pm standard deviation. Tukey test $($ alpha $=0.05)$.

\begin{tabular}{lcc}
\hline $\begin{array}{c}\text { Altura de } \\
\text { la muestra }\end{array}$ & \multicolumn{1}{c}{$\begin{array}{c}\text { Procedencia } \\
\text { local }\end{array}$} & $\begin{array}{c}\text { Procedencia } \\
\text { Valdivia-Chile }\end{array}$ \\
\hline Base $(0,3 \mathrm{~m})$ & $523,9 \pm 38 \mathrm{aB}$ & $479,7 \pm 41 \mathrm{aA}$ \\
AP $(1,3 \mathrm{~m})$ & $512,2 \pm 37 \mathrm{aA}$ & $482,3 \pm 34 \mathrm{aA}$ \\
$30 \%$ altura comercial & $546,6 \pm 46 \mathrm{abB}$ & $503,7 \pm 40 \mathrm{aA}$ \\
$50 \%$ altura comercial & $572,7 \pm 45 \mathrm{bcB}$ & $500,9 \pm 52 \mathrm{aA}$ \\
$75 \%$ altura comercial & $598,3 \pm 43 \mathrm{cB}$ & $487,4 \pm 57 \mathrm{aA}$
\end{tabular}

Letras mayúsculas se leen horizontalmente, letras minúsculas se leen verticalmente; letras iguales indican diferencias no significativas. 
Longitud de fibras. El valor medio de longitud de fibras de los 20 árboles fue de $846,9 \mu \mathrm{m}$, con un rango entre $954,9 \mu \mathrm{m}$ y $742,7 \mu \mathrm{m}$. La longitud de fibras promedio de la procedencia Valdivia-Chile resultó menor $(830,2 \mu \mathrm{m} \pm 67,4 \mu \mathrm{m})$ que la de la procedencia local $(863,6 \mu \mathrm{m} \pm 38,8 \mu \mathrm{m})$ y de una mayor variabilidad entre árboles.

El análisis estadístico indicó a la procedencia como no significativa $(P=0,1909)$, en tanto que resultó significativa la posición radial $(P<0,001)$ y la altura en el fuste $(P=0,0061)$. Asimismo, la interacción procedencia-posición fue significativa $(P<0,001)$ y su apertura (cuadro 2$)$ permitió observar que las procedencias manifestaron similar longitud de fibras en la región próxima a la corteza (90 \% de la longitud del radio), no así en la región próxima a la médula ni en la intermedia entre médula y corteza (50\% de la longitud del radio).

La interacción altura-procedencia no resultó significativa $(P=0,1327)$; así, en sentido base-ápice, la longitud de fibras varió significativamente pero bajo patrones que deberían considerarse similares entre las procedencias. La interacción altura-posición tampoco resultó significativa $(P=0,6149)$, por lo que los tejidos próximos a la médula, los intermedios y los próximos a la corteza manifestaron patrones de variación a lo largo del fuste que también deberían considerarse similares. El árbol como fuente de variación participó con el $59 \%$ en la varianza aleatoria total.

Dada la ausencia de estas interacciones, el análisis de los datos agrupados según alturas de muestreo sin discriminar procedencias ni posiciones radiales (prueba de Tukey, alfa $=0,05$ ), indicó que a lo largo del fuste la región correspondiente al $50 \%$ de la altura total fue la que se diferenció significativamente del resto por su menor magnitud en la longitud de fibras.

Relación entre los parámetros de calidad de la madera y el crecimiento. Las procedencias manifestaron diferencias en el incremento medio anual en altura total, siendo

Cuadro 2. Variación radial de la longitud de fibras $(\mu \mathrm{m})$ según las procedencias. Promedios \pm desviación estándar. Prueba de Tukey $($ alfa $=0,05)$.

Radial variation of fiber length $(\mu \mathrm{m})$ by provenances Mean \pm standard deviation. Tukey test (alpha $=0.05$ ).

\begin{tabular}{lcc}
\hline \multicolumn{1}{c}{$\begin{array}{c}\text { Posición } \\
\text { radial }\end{array}$} & $\begin{array}{c}\text { Procedencia } \\
\text { local }\end{array}$ & $\begin{array}{c}\text { Procedencia } \\
\text { Valdivia-Chile }\end{array}$ \\
\hline $\begin{array}{l}10 \% \text { de la longi- } \\
\text { tud del radio }\end{array}$ & $693,1 \pm 159 \mathrm{aB}$ & $620,6 \pm 123 \mathrm{aA}$ \\
$\begin{array}{l}50 \% \text { de la longi- } \\
\text { tud del radio }\end{array}$ & $898,4 \pm 198 \mathrm{bB}$ & $860,9 \pm 160 \mathrm{bA}$ \\
$\begin{array}{l}90 \% \text { de la longi- } \\
\text { tud del radio }\end{array}$ & $999,5 \pm 213 \mathrm{cA}$ & $1.009,2 \pm 189 \mathrm{cA}$ \\
\hline
\end{tabular}

Letras mayúsculas se leen horizontalmente, letras minúsculas se leen verticalmente; letras iguales indican diferencias no significativas. mayores en Valdivia-Chile $\left(2,6 \mathrm{~m}\right.$ año ${ }^{-1} \pm 0,3 \mathrm{~m}$ año $\left.\mathrm{o}^{-1}\right)$ que en la local $\left(2,1 \mathrm{~m}\right.$ año $\left.{ }^{-1} \pm 0,2 \mathrm{~m}_{\text {año }}{ }^{-1}\right)$. En términos de incremento medio anual en volumen comercial con corteza resultaron similares, con mayor variabilidad entre árboles en la procedencia Valdivia-Chile $\left(0,0201 \mathrm{~m}^{3} \mathrm{año}^{-1} \pm 0,007\right.$ $\mathrm{m}^{3}$ año $\left.^{-1}\right)$ que en la local $\left(0,0196 \mathrm{~m}^{3}\right.$ año $^{-1} \pm 0,003 \mathrm{~m}^{3}$ $\mathrm{año}^{-1}$ ). Asimismo, las procedencias resultaron similares en su incremento medio anual en DAP: $2,1 \mathrm{~cm}$ año $0^{-1} \pm 0,4$ $\mathrm{cm}$ año-1 para Valdivia-Chile, y $2,3 \mathrm{~cm}$ año ${ }^{-1} \pm 0,1 \mathrm{~cm}$ año $0^{-1}$ para la local. Valdivia-Chile presentó mayor variabilidad entre árboles.

La prueba de correlación indicó la ausencia de relación lineal significativa $(P>0,05)$ entre la densidad básica del árbol completo y el incremento medio anual en $\operatorname{DAP}(0,26)$, en altura $(-0,34)$ y en volumen comercial $(0,18)$. Tampoco se manifestó una relación lineal significativa $(P>0,05)$ entre la longitud de fibras del árbol completo y dichos incrementos $(0,07 ;-0,13 ; 0,13$, respectivamente).

\section{DISCUSIÓN}

Los valores promedio de densidad básica determinados en el presente trabajo están comprendidos en el rango considerado adecuado para la producción de pulpas y papeles de $400 \mathrm{~kg} \mathrm{~m}^{-3}$ a $600 \mathrm{~kg} \mathrm{~m}^{-3}$ (Downes et al. 1997) y, a excepción del mínimo indicado para la procedencia Valdivia-Chile, quedan también comprendidos entre los valores informados por otros autores en plantaciones de edades similares. Así, Muneri y Raymond (2001) en plantaciones de siete años en Australia informaron valores entre $460 \mathrm{~kg} \mathrm{~m}^{-3}$ y $570 \mathrm{~kg} \mathrm{~m}^{-3}$; Tomazello Filho (1987) de $480 \mathrm{~kg} \mathrm{~m}^{-3}$ en plantaciones de 10 años en Brasil; Miranda et al. (2001ab) en plantaciones de siete años, indicaron valores desde $442 \mathrm{~kg} \mathrm{~m}^{-3}$ hasta $450 \mathrm{~kg} \mathrm{~m}^{-3}$ en tres sitios de Portugal, y de $492 \mathrm{~kg} \mathrm{~m}^{-3}$ a $600 \mathrm{~kg} \mathrm{~m}^{-3}$ para las mismas plantaciones a los nueve años de edad. En estudios locales sobre una plantación comercial de procedencia local de nueve años de edad la densidad básica determinada fue de $531 \mathrm{~kg} \mathrm{~m}^{-3}$ (Igartúa y Monteoliva 2006).

La variación de la densidad entre árboles resulta destacada en el material estudiado. Estas diferencias fenotípicas entre los árboles han sido indicadas por algunos autores como la mayor fuente de variación en latifoliadas de porosidad difusa para la densidad básica (Downes et al. 1997). También se ha indicado que, dado el fuerte determinismo genético sobre las características estructurales del leño, es de esperar encontrar diferencias entre árboles, aun en casos donde éstos se desarrollen sobre el mismo sitio y sean contemporáneos, como es el caso en el presente estudio (Wilkes 1988). Otros autores han descrito notorias variaciones individuales de la densidad en Eucalyptus grandis (Hill) Maiden (Ferreira 1972, Brasil y Ferreira 1972, Taylor 1973) y en Eucalyptus nitens (Deane et Maiden) Maiden (Kibblewhite et al. 1998). En anteriores estudios regionales sobre el recurso de E. globulus de 35 años de 
edad, las diferencias entre los árboles resultaron poco destacadas en una estación de crecimiento y significativas en otra (Igartúa et al. 2002, 2003).

Las procedencias se diferencian en la densidad básica de su madera siendo ésta mayor en la local. También se diferencian en sus tendencias de variación interna a lo largo del fuste, la procedencia local incrementándola a partir del $30 \%$ de la altura total y Valdivia-Chile manteniéndola sin cambios a lo largo del fuste (cuadro 1). Ambos patrones han sido descritos en la literatura, donde se ha mencionado como característica general a la manifestación de un aumento de la densidad desde la base hacia el ápice (Ferreira 1972, Valente et al. 1992, Downes et al. 1997). Dentro de tal generalización, también se ha informado la disminución del valor de la densidad hasta la altura del pecho, o hasta el 15- $20 \%$ de la altura del fuste, seguida de un incremento hacia el ápice, tendencia aquí mostrada por la procedencia local (Taylor 1973, Busnardo et al. 1983, 1987, Wilkes 1988, Downes et al. 1997, Raymond y Muneri 2001). Downes et al. (1997), en su revisión de los modelos de variación de la densidad básica en el género Eucalyptus, mencionaron la ausencia de variación a lo largo del fuste como otro de los patrones informados, tal es el caso de la tendencia que manifiesta la procedencia Valdivia-Chile en el presente trabajo.

En los estudios regionales anteriores sobre la especie en masas de 35 años de edad no se evaluó la región de la base del fuste, pero a partir de la AP la densidad aumentó hacia el ápice, patrón de variación similar al encontrado en el presente para la procedencia local por cuanto también significa un aumento base-ápice de la densidad en contraposición a lo que sería la ausencia de variación (Igartúa et al. 2002, 2003).

Las procedencias no se diferencian en su longitud de fibras promedio, si bien hay una tendencia a ser mayor en la local, aunque no resulta significativa estadísticamente ni lo sería desde el punto de vista tecnológico. La longitud de fibras observada en ambas procedencias resulta algo mayor que lo informado en plantaciones de siete años por Muneri y Raymond (2001) en dos sitios de Australia (750 $\mu \mathrm{m}$ y $740 \mu \mathrm{m}$, respectivamente). Tomazello Filho (1987) encontró como valor promedio a la altura del pecho una longitud de $810 \mu \mathrm{m}$ en plantaciones de 10 años en Brasil. En cambio, Miranda et al. (2001c) indicaron valores desde $817 \mu \mathrm{m}$ a $1050 \mu \mathrm{m}$ en plantaciones de siete años de edad en tres sitios de Portugal.

Para el género y la especie se ha informado, dentro de una variación a lo largo del fuste aceptada como pequeña, la disminución de la longitud de fibras en sentido base-ápice (Wilkes 1988, Valente et al. 1992), así como también la falta de influencia de la altura del árbol (Taylor 1973). También se ha descrito el incremento leve de la longitud de fibras desde la base hacia regiones comprendidas, según diferentes autores, en el rango 15 a $35 \%$ de la altura del fuste, seguido de su decrecimiento hacia el ápice (Bisset y Dadswell 1949, Wilkes 1988, Ridoutt y
Sands 1993, Ridoutt y Sands 1994, Jorge 1996, Downes et al. 1997, Jorge et al. 2000, Muneri y Raymond 2001, Kojima et al. 2009).

Este último patrón de variación de la longitud de fibras a lo largo del fuste resulta similar al que manifiestan las dos procedencias aquí estudiadas. Ambas muestran tendencias que deberían considerarse similares entre sí, con un descenso significativo hacia el ápice, particularmente entre el 30 y el $50 \%$ de la altura total del fuste. En estudios anteriores sobre masas maduras de la especie se evaluó el fuste entre la AP y el $60 \%$ de la altura total y la variación descrita fue de un leve y no significativo ascenso hacia el ápice (Igartúa et al. 2000, 2002, 2003).

En referencia a la variación radial en la longitud de fibras, ambas procedencias manifiestan su incremento desde la médula hacia la región de la corteza, tendencia que se ha descrito en el género y la especie, y se ha considerado como la principal fuente de variación (Brasil y Ferreira 1972, Taylor 1973, Tomazello Filho 1987, Wilkes 1988, Valente et al. 1992, Jorge 1996, Jorge et al. 2000, Igartúa et al. 2000, 2003, Kojima et al. 2009). En el marco de tal incremento, sin embargo, las procedencias local y ValdiviaChile muestran diferencias entre la longitud de fibras de sus tejidos más internos (hasta el $50 \%$ de la longitud del radio) siendo ésta mayor en la procedencia local, e iguales sus valores en la región próxima a la corteza por lo que estos patrones de incremento radial deberían considerarse diferentes entre sí (cuadro 2).

En dirección radial, a cualquier altura del fuste, la longitud de fibras aumenta desde la médula a la periferia. Este comportamiento, que se manifiesta como una interacción altura-posición radial no significativa en el presente trabajo, ha sido ampliamente informado en el género desde los trabajos de Bisset y Dadswell (1949) y, en la especie, en árboles de diferentes edades entre los 7 y 35 años (Downes et al. 1997, Igartúa et al. 2000, 2002, Kojima et al. 2009).

Las diferencias significativas que las procedencias estudiadas manifiestan en su densidad básica podrían adjudicarse a causas genéticas entendiendo que la característica de ser coetáneas, desarrolladas sobre un mismo sitio y bajo una misma conducción silvicultural, estaría disminuyendo la componente ambiental de esta manifestación fenotípica. Sin embargo, más investigación debería ser desarrollada bajo esta hipótesis.

En el marco del análisis realizado no se encuentra correlación significativa entre los incrementos medios individuales (en DAP, en altura total y en volumen comercial) y la densidad básica de la madera del árbol completo, tanto como entre dichos incrementos y la longitud de fibras del árbol completo. El material experimental se manifiesta en un rango acotado de variación en las variables epidométricas y, del mismo modo, podría considerarse acotado el rango de variación entre las estimaciones de las variables de calidad de la madera para los árboles completos. Esta característica de los datos puede estar impidiendo la detección de una 
relación lineal significativa. Se considera necesario continuar indagando en las diferentes calidades de sitio que la región ofrece, en las diferentes edades que es posible reconocer en el recurso, en la diversidad que podría esperarse encontrar bajo la denominada procedencia local y en la ampliación de la tarea inventarial a nivel de las poblaciones.

Principales resultados. La madera del recurso regional de E. globulus muestra, en general, magnitudes y patrones de variación interna para la densidad básica y la longitud de fibras que se corresponden con lo informado en otros estudios en la especie. Las procedencias local y ValdiviaChile manifiestan destacadas diferencias tanto en densidad básica como en su tendencia de variación a lo largo del fuste. La primera de ellas presenta mayor magnitud de esta propiedad, relevante desde el punto de vista tecnológico, y la aumenta en sentido base-ápice, en tanto que ValdiviaChile manifiesta ausencia de tal variación. La longitud de fibras y los patrones de variación a lo largo del fuste se consideran similares entre ambas procedencias. La mayor fuente de variación interna para la longitud de fibras es la edad fisiológica del leño captada con el muestreo radial. La variabilidad entre árboles dentro de procedencia es una destacada fuente de variación para estos dos parámetros de calidad de la madera. Las procedencias se diferencian por su IMA en altura total a los siete años de edad (mayor en Valdivia-Chile), no así por sus IMA en diámetro y en volumen comercial. No se encuentran correlaciones lineales entre ninguno de estos incrementos medios y las dos propiedades de la madera evaluadas.

\section{CONCLUSIONES}

Como materia prima para el sector industrial celulósico papelero, la madera de la procedencia Valdivia-Chile, a los siete años de edad, presenta propiedades en términos de densidad básica y de longitud de fibras que la califican como de menor aptitud papelera frente a la procedencia local, a la que tampoco supera en crecimiento medio en volumen comercial.

\section{REFERENCIAS}

Bisset WJ, HE Dadswell. 1949. The variation of fibre length within one tree of Eucalyptus regnans, F. y M. Australian Forestry 13(2): 86-96.

Brasil MAM, M Ferreira. 1972. Variaçao da densidade básica e das características das fibras em Eucalyptus grandis Hill ex Maiden ao nivel do DAP. IPEF 5: 81-90.

Busnardo CA, JV Gonzaga, CE Foelkel, C Diaz, S Menochelli. 1983. Em busca da qualidade ideal da madeira do eucalipto para producao de celulose. III. Importancia da altura de amostragem para availacao da densidade basica media da arvore. In III Congresso Latinoamericano de Celulosa y Papel, Brasil. 17 p.
Busnardo CA, JV Gonzaga, CE Foelkel, S Menochelli. 1987. Em busca da qualidade ideal da madeira do eucalipto para producao de celulose. IV. Altura ideal de amostragem para availacao da densidade media para arvore de E. grandis. In XX Congresso Anual da ABCP, Brasil. 16 p.

Cailliez F. 1980. Estimación del Volumen Forestal y predicción del rendimiento. Estudio FAO: Montes 22/1. Roma, Italia. Organización de las Naciones Unidas para la Agricultura y la Alimentación FAO. 89 p.

Downes GM, IL Hudson, CA Raymond, AJ Dean, AJ Michell, LR Schimleck, R Evans, A Muneri. 1997. Sampling Eucalypts for wood and fibre properties. Melbourne, Australia. CSIRO Publishing. $132 \mathrm{p}$.

Ferreira M. 1972. Variaçao da densidade básica da madeira de povamentos comerciais de Eucalyptus grandis Hill ex Maiden nas idades de 11, 12, 13, 14 e 16 anos. IPEF 4: 65-89.

Foelkel C. 1998. Eucalypt wood and pulp quality requirements oriented to the manufacture of tissue and printing \& writing papers. In $52^{\text {nd }}$ Annual General Conference, Brisbane, Australia. Anais APPITA. 6 p.

Golfari L. 1957. Distribución y ecología de algunos eucaliptos cultivados en la Argentina. In Primeras Jornadas Argentinas del Eucalipto. 4-10 noviembre de 1957. AfoA. Buenos Aires, Argentina. $12 \mathrm{p}$.

Igartúa DV, SM Rivera, MG Monterubbianesi, S Monteoliva, MS Villegas, S Carranza, S Farina. 2000. Calidad del leño en E. globulus ssp. globulus. I-Variación de la densidad básica y la longitud de fibras en una estación del sudeste de la provincia de Buenos Aires, Argentina. In Congreso Iberoamericano de Investigación en Celulosa y Papel. Octubre de 2000, Misiones, Argentina. 20 p.

Igartúa DV, S Monteoliva, MG Monterubbianesi, MS Villegas. 2002. Calidad del leño en Eucalyptus globulus ssp. globulus: II. Variaciones en la densidad básica y la longitud de fibras en Lobería, provincia de Buenos Aires, Argentina. Revista de la Facultad de Agronomía de La Plata 105(1): 29-39.

Igartúa DV, S Monteoliva, MG Monterubbianesi, y MS Villegas. 2003. Sampling at breast height (bh) level of basic density and fibre length for Eucalyptus globulus ssp. globulus for parameter prediction of the whole-tree. IAWA Journal (24)2: 173-184.

Igartúa DV, S Monteoliva. 2006. El Eucalyptus globulus en Argentina: potencialidad del sudeste de la provincia de Buenos Aires para la producción de materia prima fibrosa. In IV Congreso Iberoamericano de Investigación en Celulosa y Papel. Santiago y Valdivia-Chile. 23 al 27 de octubre de 2006.8 p.

Jane FW. 1970. The structure of wood. London, England. $2^{\text {nd }}$ ed. Adam and Charles Black eds. 478 p.

Jorge F. 1996. Variabilidad anatómica da madeira da Eucalyptus globulus Labill. In XV Congresso Nacional Tecnicelpa. Centro de Cultura e Congresos de Aveiro. Lisboa, Portugal. 9 p.

Jorge F, T Quilhó, H Pereira. 2000. Variability of fibre length in wood and bark in Eucalyptus globulus. IAWA Journal 21(1): 41-48.

Kibblewhite PR, M Ridell, CJA Shelbourne. 1998. Kraft fibre and pulp qualities of 29 trees of New Zealand grown Eucalyptus nitens. Appita Journal 51(2): 114-121.

Kojima M, H Yamamoto, M Yoshida, Y Ojio, K Okumura. 2009. Maturation property of fast-growing hardwood plantation species: a view of fiber length. Forest Ecology and Management 257: 15-22. 
Kuhel RO. 2003. Diseño de experimentos. México DF, México. Thomson-Learning. $666 \mathrm{p}$.

Miranda I, MH Almeida, H Pereira, 2001a. Influence of provenance, subspecies and site on wood density in Eucalyptus globulus Labill. Wood and Fiber Science 33(1): 9-15.

Miranda I, MH Almeida, H Pereira. 2001b. Provenance and site variation of wood density in Eucalyptus globulus Labill at harvest age and its relation to a non-destructive early assessment. Forest Ecology and Management 149: 235-240.

Miranda I, MH Almeida, H Pereira. 2001c. Variation of fibre biometry in different provenances of Eucalyptus globulus Labill. Appita Journal 54(3): 272-280.

Moschini RC, HA Conti, M Alonso, J Rodríguez Traverso, V Nakama, A Alfieri. 2000. Delimitación de áreas de aptitud climática para el cultivo de los eucaliptos en la región pampeana. SAGPyA Forestal 15: 2-11.

Muneri A, CA Raymond. 2001. Non-destructive sampling of E. globulus and E. nitens for wood properties. II. Fibre length and coarseness. Wood Science and Technology 35: 41-56.

Prodan M, R Peters, F Cox, P Real. 1997. Mensura forestal. San José, Costa Rica. IICA. 586 p.

R Development Core Team. 2007. R: A language and environment for statistical computing. R Foundation for Statistical Computing, Vienna, Austria. ISBN 3-900051-07-0, URL http://www.R-project.org.

Ramírez M, J Rodríguez, C Balocchi, M Peredo, JP Elissetche, R Mendonca, S Valenzuela. 2009. Chemical composition and wood anatomy of Eucalyptus globulus clones: variations and relationships with pulpability and handsheet properties. Journal of Wood Chemistry and Technology 29(1): 43-58.
Raymond CA, A Muneri. 2001. Nondestructive sampling of Eucalyptus globulus and Eucalyptus nitens for wood properties. I. Basic density. Wood Science and Technology 35: 27-39.

Ridoutt BG, R Sands. 1993. Within-tree variation in cambial anatomy and xylem cell differentiation in Eucalyptus globulus. Trees 8: 18-22.

Ridoutt BG, R Sands. 1994. Quantification of the processes of secondary xylem fibre development in Eucalyptus globulus at two height levels. IAWA Journal 15(4): 417-424.

Taylor F. 1973. Anatomical wood properties of South African grown Eucalyptus grandis. South African Forestry Journal 84: 20-24.

Tomazello Filho M. 1987. Variaçao radial da densidade básica e da estrutura anatomica da madeira do Eucalyptus globulus, E. Pellita e E. acmenioides. IPEF 36: 35-42.

Torres U, S Rodríguez. 1991. Pulpaje kraft con trozas de Eucalyptus globulus Labill. en diferentes diámetros. Bosque 12(2): 65-68.

Valente CA, A Mendes de Sousa, FP Furtado, AP de Carvalho. 1992. Improvement program for Eucalyptus globulus at PORTUCEL: Technological component. Appita 45(6): 403-407.

Wilkes J. 1988. Variations in wood anatomy within species of Eucalyptus. IAWA Bulletin n.s. 9(1): 13-23.

Zobel BJ. 1997. Genetics of Wood-An Overview. In Zhang S, R Gosselin, G Chauvet eds. Timber management toward wood quality and end-product value. CTIA/IUFRO International Wood Quality Workshop. Quebec, Canada. August 18-22, 1997. $6 \mathrm{p}$.

Recibido: 11.11.09

Aceptado: 28.04.10 